\title{
\begin{tabular}{|l|l|l|l|l|}
\hline $\mathrm{M}$ & $\mathrm{R}$ & $\mathrm{S}$ & Internet Journal of & Nitride Semiconductor Research \\
\hline
\end{tabular}
}

Volume 1, Article 27

\section{Temperature distribution in the chamber used for crystal growth of GaN under high pressure of nitrogen}

\author{
S. Krukowski \\ High Pressure Research Center
}

This article was received on June 4, 1996 and accepted on October 30, 1996.

\begin{abstract}
The theoretical analysis of high pressure influence on the conditions of crystal growth of GaN is presented. High pressure influence on the transport and equilibrium properties of nitrogen is estimated using scaling approach. Nitrogen gas properties are used in the finite element calculation of the thermal conditions in the high pressure chamber. The temperature distribution during GaN growth in the vertical temperature-gradient configuration is obtained.
\end{abstract}

\section{Introduction}

Recent progress in the construction of nitride based optoelectronic devices has been achieved by application of heteropitaxial growth techniques [1] [2]. Nitride layers were grown sapphire [3] as substrate and despite large lattice mismatch with GaN both LED and lasers were constructed [4]. At the present a nitride based laser is able to work only for a limited time. In order to extend the work period of the laser, the quantum well structures should be improved.

Heteroepitaxial technologies will always suffer from the differences in the properties of substrate and the layers. Therefore development of homoepitaxial growth techniques is the best way to achieve fast progress in the laser technology. That requires single GaN crystals of substrate quality and the sizes of order of $1 \mathrm{~cm}$. Growth of GaN single crystals is difficult because it requires high activity of nitrogen and high temperatures [5].

Gallium nitride single crystals can be grown by ammonia [6] or by high pressure nitrogen methods [7] [8]. Since $\mathrm{NH}_{3}$ is unstable in the temperatures above $1100^{\circ} \mathrm{C}$, the ammonia method suffers from low solubility of nitrogen in the liquid gallium. On the contrary the high $\mathrm{N}_{2}$ pressure method allows to extend the temperature range of the growth to $1500^{\circ} \mathrm{C}$ for nitrogen pressures close to $20 \mathrm{kbar}$. So high pressure changes equilibrium and transport properties of nitrogen drastically. On the contrary, the physical properties of liquid gallium remain essentially unchanged. For high pressure the volume of the chambers is limited which leads to very steep temperature gradients close to the growth zone. Therefore the control of the temperature in the high pressure chamber is of a primary importance to the design of high pressure growth apparatus.

In this paper we will give an outline of the thermodynamic properties of nitrogen in pressures up to $20 \mathrm{kbar}$ and the temperatures up to $1600^{\circ} \mathrm{C}$. Then we employ, finite element code FIDAP [9] to obtain the temperature inside high pressure chamber. The calculations of one-zone furnace are shown. These results are analysed and compared with the data obtained in high pressure experiments.

\section{Nitrogen properties under high pressure}

High pressure of the nitrogen drastically changes its physical properties. The most important effect is the increase of the density of the gas. This change of the nitrogen properties is largest in low temperatures. In particular strong repulsion of the atomic cores leads to the steep increase of nitrogen activity. It also enhances the direct transfer of the energy leading to the increase of thermal conductivity and shear viscosity. 


\subsection{Density and thermal expansion coefficient}

The $p$-T-r equation of state of nitrogen was critically assessed by Jacobsen et al for the interval region extending from the triple point to the pressure up to $10 \mathrm{kbar}$ and temperatures up to $2000 \mathrm{~K}$ [10]. The errors were smaller than $0.3 \%$ for the entire interval outside the critical region. The calculated density and thermal expansion coefficient obtained from extension of Jacobsen equation of state for higher pressures and temperatures are presented in Figure 1.

\subsection{Activity}

The excess of free energy DG can be translated into the activity of the nitrogen a as follows:

$$
\Delta \mathrm{G}=\mathrm{RT} \ln \mathrm{a}
$$

Due to the repulsion of nitrogen atomic cores, free energy of nitrogen at higher pressure increases, especially in low temperatures. This increase is used in high pressure experiments to extend the stability range of GaN and plays crucial role in high pressure growth of gallium nitride. The nitrogen activity is presented in Figure 2.

\subsection{Heat capacity at constant pressure}

Heat capacity belongs to the properties which are least affected by high pressures. The kinetic degrees of freedom contribution is determined by the equipartition principle. The potential degrees of freedom contribution is affected only by anharmonicity of the interaction potential close to the minimum. Therefore a small increase of the heat capacity for higher pressures is related to motion in the potential in the other molecules. On the other hand, the increase of the heat capacity at higher temperatures is due to the internal degrees of freedom of the nitrogen molecules that are induced for high temperatures.

\subsection{Thermal conductivity}

High pressure experimental investigations of nitrogen thermal conductivity were limited to pressures not higher than $1 \mathrm{kbar}$ and the temperatures up to $1200 \mathrm{~K}$ [11] [12]. For higher temperatures nitrogen thermal conductivity was measured only for low pressures. The most successful extrapolation of thermal conductivity uses division into low density viscosity that accounts for the kinetic part of the energy transfer and the density dependent term which represents the transfer via potential energy [13]. The physical conditions of our interests covers the same interval of the nitrogen densities, as those known from low temperature measurements [11][12] with the extension to higher temperatures. Therefore we expect that the expansion

$$
\lambda(Z, \rho)=\lambda d(T)+\sum_{i=1} \mathrm{C}_{i} \rho^{i}
$$

is valid for the range of the densities of our interest. The density expansion represents the excess thermal conductivity with the precision better than $3 \%$ in the densities up to $1 \mathrm{~kg} / \mathrm{m}^{3}$. The zero density contribution $\mathrm{I}_{0}(\mathrm{~T})$ can be represented by the series:

$$
\lambda_{d} \lambda=\sum_{i=1} A_{i} \ln \left(\frac{\mathrm{kT}}{s}\right)
$$

This expansion used scaling hypothesis of the temperature dependence of the collision integral crossection for the nitrogen at high temperatures. This contribution is remarkably well represented with formula (3), it recovers the experimental data with the precision better than $2 \%$ for the temperature range up to $2400 \mathrm{~K}$ [14]. Using these approach we obtained results for higher pressures which are presented in Figure 4. .

The transition from potential to collisional energy transfer leads to the minimum known as Enskog minimum. The minimum shifts towards higher temperatures for higher pressures. For higher pressures the potential term dominates therefore the transition moves into higher kinetic energy values (i.e. higher temperatures). 


\subsection{Viscosity}

The temperature dependence of the shear viscosity is presented in Figure 5. The zero density viscosity and thermal conductivity are related by heat capacity dependent factor:

$$
r d(7)=\frac{\lambda_{d}(7) A}{C_{(J)}}
$$

where $\mathrm{M}$ - is the molecular mass and $\mathrm{C}_{\mathrm{V}}$ - heat capacity under constant volume, which weakly depends on the temperature. The excess viscosity can be expressed in power of the density. The resulting temperature dependence shows again the Enskog minimum, which is shifted towards higher temperatures for higher pressures.

\subsection{Summary}

From these properties one can deduce that the effect of the pressure will have different influence in the intermediate and very high pressure. For the intermediate pressures, the convective energy transfer increases due to sharp increase of the density and the heat capacity. For very high pressure the increase of viscosity slows the convective motion exerting stabilizing influence on the gas system. Similar influence has sharp decrease of the volume expansion coefficient. Furthermore, the increase of the conductivity reduces temperature gradients requiring higher heat power of the furnace which poses more stringent demands for the heat production in the system. The increase of the density and heat capacity plays minor role and will not reduce the convective transport in a significant way.

A radiative heat transfer is not affected by the pressure changes. Therefore we assumed that this radiative heat exchange is not affected by the pressure in all our simulations.

\section{Finite element calculations}

In the calculation we have used finite element code FIDAP [9]. The code is solving entire set of Navier-Stokes equations for liquid part of the system and the thermal conductivity equation for the solid part. In the nitrogen part the radiative heat exchange was also accounted for. The surfaces were assumed to be perfectly black bodies which is good approximation for the temperatures higher than $1200^{\circ} \mathrm{C}$. The gallium liquid properties were assumed to be pressure independent. Similar assumption has been used for the heat conductivity in the solid part of the system.

The high pressure apparatus in the vertical configuration has been calculated. It consists of three tube steel chamber of the internal diameter of $40 \mathrm{~mm}$ and the length of $203 \mathrm{~mm}$. The water cooling is used to keep the temperature of the chamber close to the equilibrium temperature. The temperature of the chamber is monitored during the entire growth process. The variation of the temperature do not exceed $10^{\circ} \mathrm{C}$.

The heater is in form of graphite spiral. In these calculations the heater is represented by a uniform heat source of the total power equal to $1500 \mathrm{~W}$ which is equal to the electrical power used in the growth experiment. The temperature distribution has cylindrical symmetry, therefore in Figure 6 we present the cut though the simplified design of the chamber and the isotherm of obtained in the calculation.

The calculation procedure used the nonuniform grid, defined so that the maximal density is in the region of expected steep temperature gradients. The total number of the grid points was close to $2.5{ }^{*} 10^{4}$. The solution vector has been attributed a norm which is a sum over all grid points. The iteration procedure was repeated until relative change of the norm of the temperature field was smaller than $10^{-5}$ and the residue norm was smaller than 0.1 . The change of the grid density in the region of the steepest gradients has not changed the solution by more than 0.001 of the obtained values.

The results were obtained in the calculation that include or neglects radiative heat exchange. The radiative contribution depends on the detail design of the apparatus. In particular that strongly depends on the approximation of the spiral by the heat source of the shape of the uniform tube. Depending on the details of the exchange between spiral and the internal part of the crucible, the radiation effects can change the temperature of the gallium part by $25 \%$. 
Results of the calculation that include the radiative effects were compared with the temperature measurements in the high pressure growth experiments. The temperature has been measured at the three points of the crucible, close to liquid $\mathrm{Ga}$. The difference between the temperature measured in the experiments and those obtained in our calculation was not higher than $10 \%$.

\section{Summary}

The results obtained in this work can be summarised as follows:

- high pressure (of order of $20 \mathrm{kbar}$ ) stabilizes the system - it reduces the thermal gradients and slows down the convective motion of the gas,

- radiative energy transfer plays important role in the heat transfer in high pressure crystal growth of GaN,

- scaling hypothesis based methods are capable to estimate the equilibrium and transport properties of nitrogen in pressures up to $20 \mathrm{kbar}$ and temperatures up to $2000 \mathrm{~K}$,

- computer methods are able to characterize the temperature distribution in high pressures up to $20 \mathrm{kbar}$ with the precision better than $10 \%$.

\section{Acknowledgement}

The research reported in this work has been supported by Polish Committee for Scientific Research grant no. 7 $783495 \mathrm{C} / 2399$.

\section{References}

[1] I. Akasaki, H. Amano, Optoelectr. Dev. Tech. 7, 49 (1992).

[2] S. N. Mohammad, A. A. Salvador, H. Morkoç, Proc. IEEE 83, 1306-1355 (1995).

[3] S. Nakamura, T. Mukai, M. Senoh, Jpn. J. Appl. Phys. 30, L1998 (1991).

[4] S. Nakamura, M. Senoh, S. Nagahama, N. Iwasa, T. Yamada, T. Matsushita, H. Kiyoku, Y. Sugimoto, Jpn. J. Appl. Phys. 35, L217-L220 (1996).

[5] JH Edgar, (Editor), Properties of Group III Nitrides (Electronic Materials Information Service (EMIS), London, 1994).

[6] D. Elwell, M. M. Elwell, Prog. Cryst. Growth and Charact. 17, 53-78 (1988).

[7] S. Porowski, I. Grzegory, J. Jun, in High Pressure Chemical Synthesis, Edited by: J. Jurczak, B. Baranowski , (Elsevier, Amsterdam, 1989) 21.

[8] I. Grzegory, S. Krukowski, Phys. Scr. T39, 242-249 (1991).

[9]Fidap User Manual, Revision 7. 5 (Fluid Dynamics International, 1993)

[10]R. T. Jacobsen, R. B. Stewart and M. Jahangiri, J. Phys. Chem. Ref. Data 15, 735(1986)

[11]W. B. Streett, "Properties of fluids at high pressure", in High Pressure Technology, edited by I. L. Spain and J. Paauwe, (Dekker, N. York 1977) 425-475

[12]B. Le Neindre, Int. J. Heat Mass Transfer 15, 1 (1971)

[13]E. McLaughlin,"Theory of Thermal Conductivity of Fluids", in Thermal Conductivity, edited by R. P. Tye, (Academic, London 1969) 1-64.

[14]K. Stephen and R. Krauss, A. Laesecke, J. Phys. Chem. Ref. Data 16 (1987) 993 


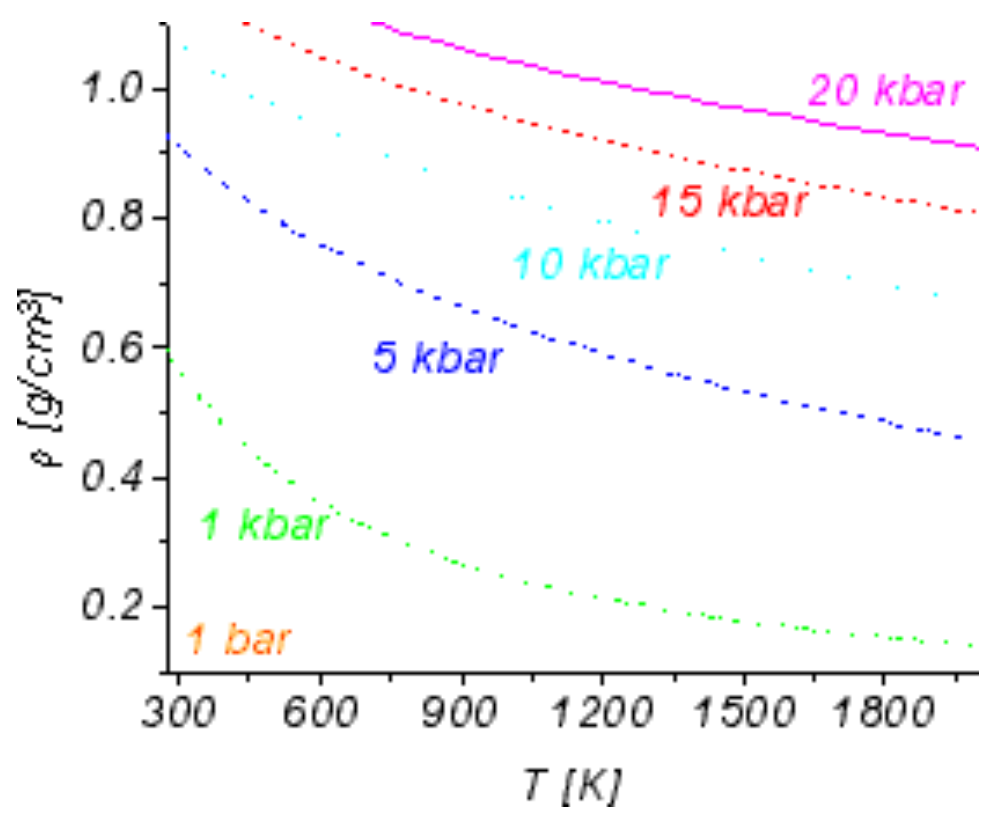

Figure 1a. High pressure isobars of nitrogen density.

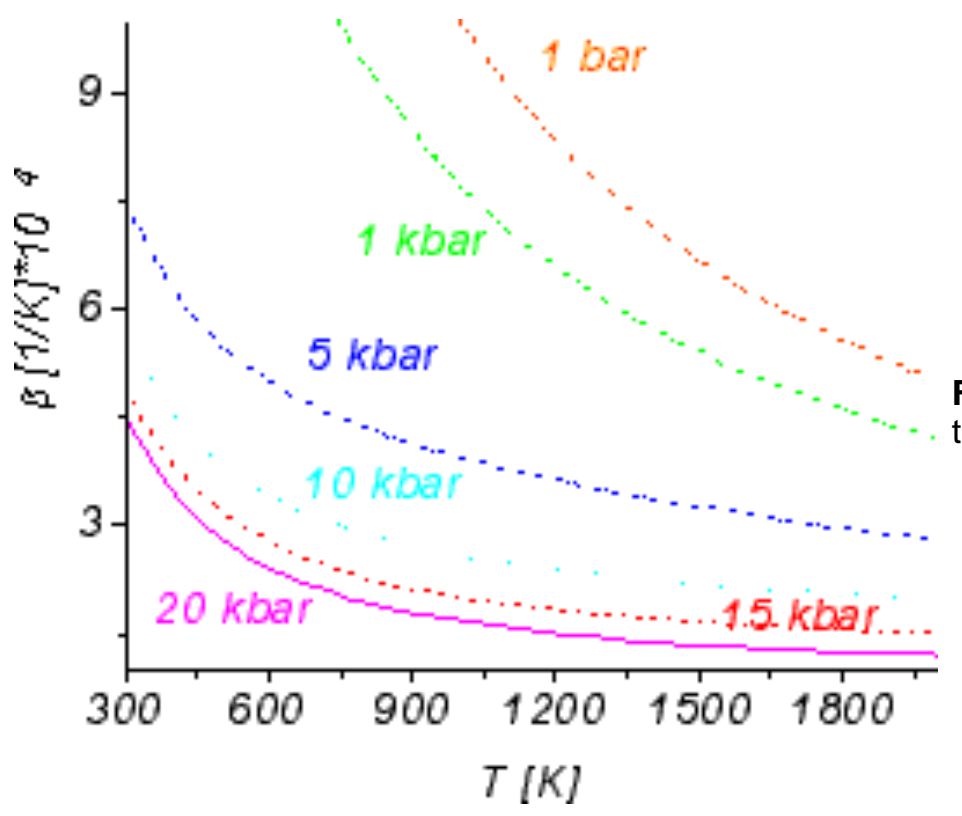

Figure 1b. High pressure isobars of nitrogen thermal expansion coefficient. 


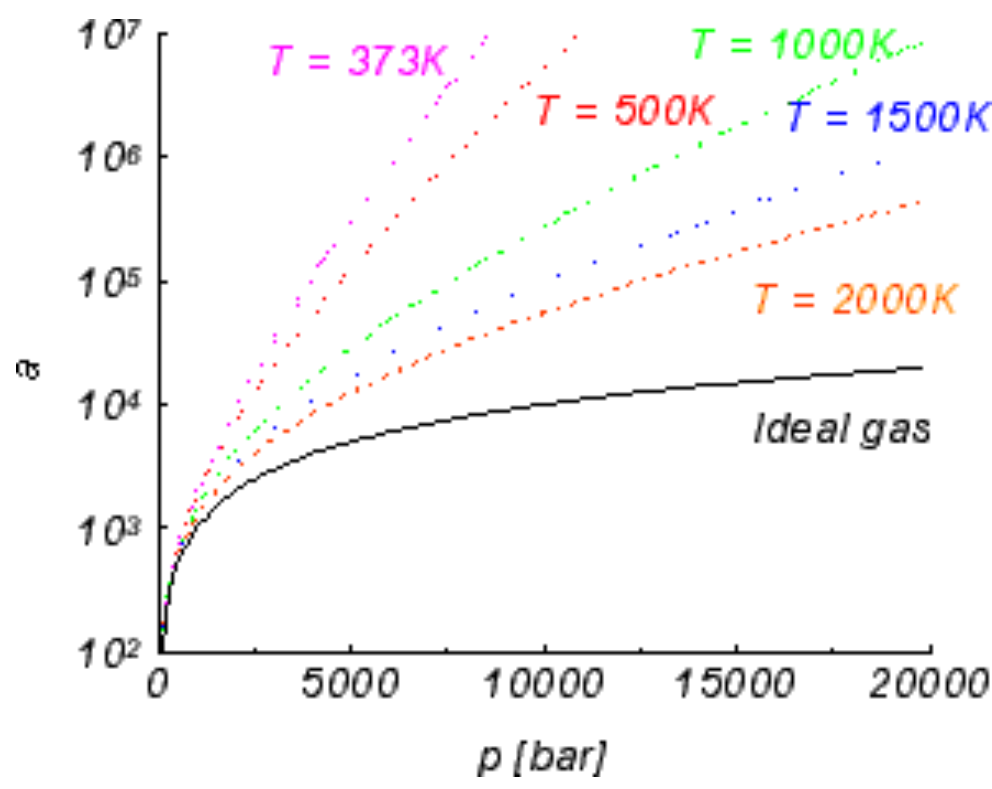

Figure 2. Nitrogen activity for several temperatures.

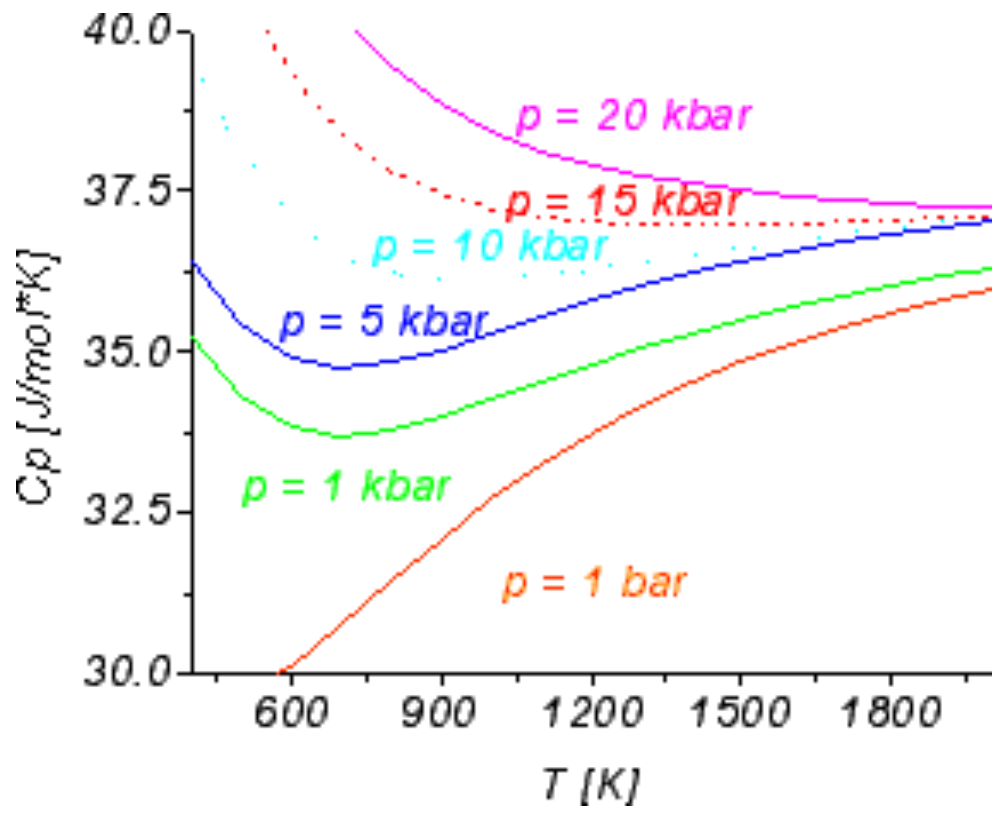

Figure 3. Heat capacity of nitrogen in high pressures.

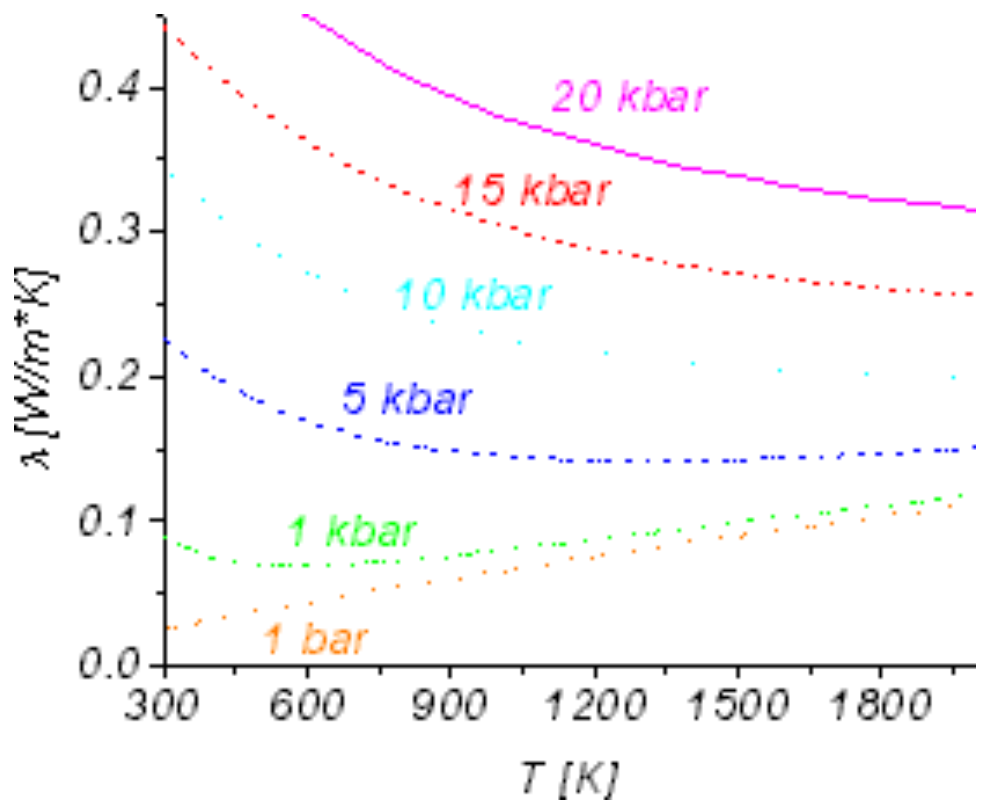

Figure 4. Thermal conductivity of nitrogen under high pressure 


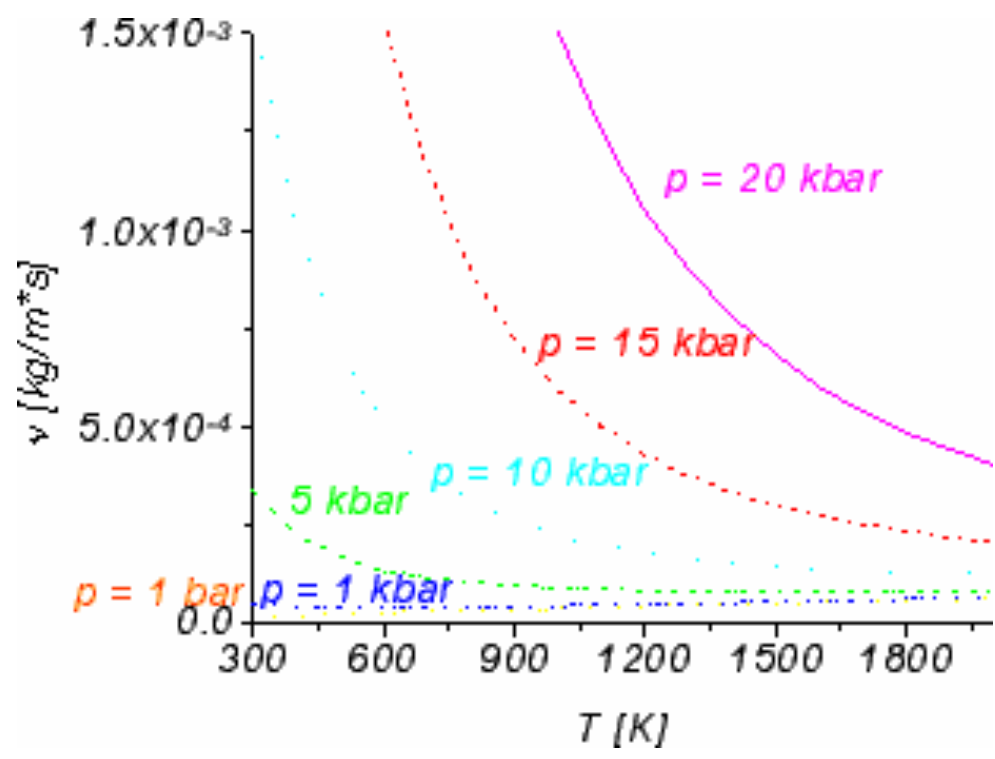

Figure 5. Viscosity of nitrogen under high pressure.
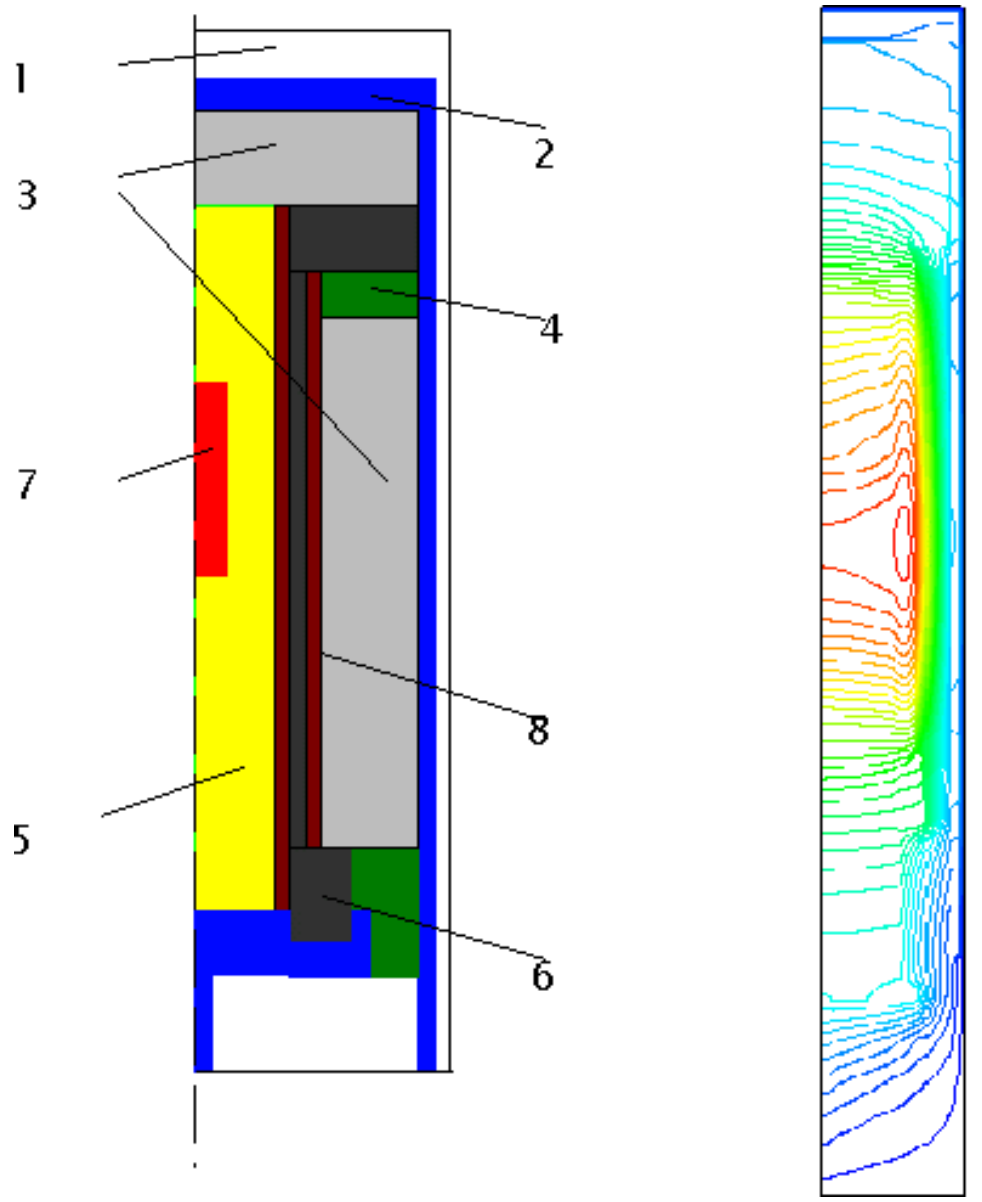

Figure 6. Schematic representation of one-zone furnace design; 1 - $\mathrm{N}_{2}$, 2 - steel, 3 - thermal isolation, 4 - pyrophillite, 5 - boron nitride, 6 graphite, 7 - $\mathrm{Ga}$ (liquid), $8-\mathrm{Al}_{2} \mathrm{O}_{3}$; the results of the calculations.

(C) 1996-1997 The Materials Research Society

\begin{tabular}{|l|l|l|l|l|}
\hline$M$ & $R$ & $S$ & Internet Journal of & Nitride Semiconductor Research \\
\hline
\end{tabular}

